\title{
Primer registro del género Pseudohydnum (Exidiaceae, Auriculariales, Basidiomycota) para Cuba
}

\section{Acta Botanica Mexicana}

\section{First record of the genus Pseudohydnum (Exidiaceae, Auriculariales, Basidiomycota) for Cuba}

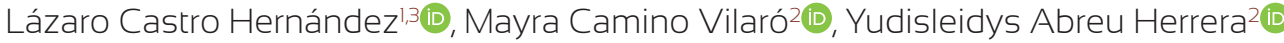

\section{Resumen:}

Antecedentes y Objetivos: El género Pseudohydnum se caracteriza por desarrollar basidiomas gelatinosos con himenóforo hidnoide, fragmobasidios longitudinalmente divididos y basidiosporas de subglobosas a elipsoides. A nivel mundial posee una amplia distribución, aunque no se registra para la micobiota cubana. El presente trabajo tuvo como objetivo reportar la presencia del género Pseudohydnum y particularmente a $P$. gelatinosum, en Cuba.

Métodos: Los especímenes fueron recolectados en la provincia de Mayabeque, Cuba. Se registraron sus características morfológicas y datos de campo; posteriormente fueron depositados en la colección micológica del herbario HAC, del Instituto de Ecología y Sistemática, perteneciente al Ministerio de Ciencia, Tecnología y Medio Ambiente de Cuba.

Resultados clave: Se registra por primera ocasión el género Pseudohydnum en Cuba, representado por P. gelatinosum. Se brindan datos de la especie, así como fotos macro y microscópicas de algunas de sus estructuras, para comparar con otras especies del género.

Conclusiones: Se amplía el área de distribución de Pseudohydnum gelatinosum y se reafirma la importancia de continuar realizando expediciones en sitios donde no existen muestreos micológicos previos.

Palabras clave: macrohongos, Mayabeque, micobiota cubana.

\section{Abstract:}

Background and Aims: The genus Pseudohydnum is characterized by developing gelatinous basidiomas with hydnoid hymenophore, longitudinally divided phragmobasidia, and subglobose to ellipsoid basidiospores. It has a globally broad distribution, although it is not registered for the Cuban mycobiota. The present work aimed to report the presence of the genus Pseudohydnum and particularly P. gelatinosum in Cuba.

Methods: The specimens were collected in the Mayabeque province, Cuba. Their morphological characteristics and field data were recorded, followed by deposition in the mycological collection of the HAC herbarium, of the Ecology and Systematics Institute, belonging to the Ministry of Science, Technology and Environment of Cuba.

Key results: The genus Pseudohydnum is recorded for the first time in Cuba, represented by P. gelatinosum. Data on the species are provided, as well as macro- and microscopic photos of some of its structures, to compare with other species of the genus.

Conclusions: The distribution area of Pseudohydnum gelatinosum is expanded and the importance of continuing to carry out expeditions in places where there are no previous samplings by mycologists is reaffirmed.

Key words: Cuban mycobiota, macrofungi, Mayabeque.

${ }^{1}$ Instituto de Ciencia Animal, carrera central km 471/2, Apdo. postal 24, San José de las Lajas, 32700 Mayabeque, Cuba.

2Instituto de Ecología y Sistemática, Carretera Varona 11835 e/ Oriente y Lindero, La Habana 19, 11900 Boyeros, La Habana, Cuba.

${ }^{3}$ Autor para la correspondencia: Ikstro96@gmail.com
Recibido: 16 de septiembre de 2021 Revisado: 19 de octubre de 2021.

Aceptado por Rosario Redonda-Martínez: 7 de diciembre de 2021.

Publicado Primero en línea: 12 de enero de 2022.

Publicado: Acta Botanica Mexicana 129(2022).

(c) (1) (5) Este es un artículo de acceso abierto By na bajo la licencia Creative Commons 4.0 Atribución-No Comercial (CC BY-NC 4.0 Internacional).
Citar como: Castro Hernández, L., M. Camino Vilaró y Y. Abreu Herrera. 2022. Primer registro del género Pseudohydnum (Exidiaceae, Auriculariales, Basidiomycota) para Cuba. Acta Botanica Mexicana 129: e1975. DOI: https://doi.org/10.21829/ abm129.2022.1975

e-ISSN: 2448-7589 


\section{Introducción}

El género Pseudohydnum Karst. (Exidiaceae), tipificado con P. gelatinosum (Scop.) Karst, se caracteriza por basidiomas gelatinosos lateralmente estipitados, con himenóforo hidnoide, fragmobasidios longitudinalmente divididos y basidiosporas de subglobosas a elipsoides, lisas. Incluye tres especies: P. brunneiceps Chen, Su \& Zhang, P. translucens Lloyd y $P$. gelatinosum. Las dos primeras solamente se han registrado de sus localidades tipo en Japón y China, respectivamente (Chen et al., 2020); mientras que $P$. gelatinosum se encuentra en algunas regiones de Europa (McNabb, 1964), Asia (Dai et al., 2009; Bera et al., 2018), Oceanía (GBIF, 2021), Norteamérica (Raymundo et al., 2012), Centro y Sudamérica (Lowy, 1971; Niveiro y Popoff, 2011), y en el caso particular del Caribe solamente en Jamaica y Puerto Rico (Lowy, 1971; Minter et al., 2001; GBIF, 2021).

En una expedición a la provincia de Mayabeque (Cuba) fueron recolectados ejemplares de Pseudohydnum los cuales se identificaron como $P$. gelatinosum, especie no documentada previamente para la micobiota cubana (Minter et al., 2001). Por lo tanto, el objetivo del presente trabajo es reportar la presencia del género Pseudohydnum y en particular de P. gelatinosum, en Cuba. Además, se proporciona una descripción detallada y fotos de estructuras macro y microscópicas del material estudiado.

\section{Materiales y Métodos}

\section{Área de estudio}

Los especímenes fueron recolectados en un bosque de vegetación secundaria en las cercanías del kilómetro 4.5 de la carretera al poblado de Casiguas (22 $57^{\prime} 51.0^{\prime \prime} \mathrm{N}$, $82^{\circ} 01^{\prime} 11.1^{\prime \prime}$ ), perteneciente al municipio San José de las Lajas (Mayabeque, Cuba). El área se localiza a 148 m s.n.m., en la ecorregión de bosques húmedos, donde se encuentran pequeños arroyos con sustrato rocoso (Olson et al., 2001). La localidad de colecta se representa en un mapa elaborado con ArcGis v. 10.2 (ESRI, 2013) (Fig. 1).

\section{Estudio de los especímenes}

Se tomaron datos y fotografías con un teléfono celular LG G4 (LG Electronics; Seúl, Corea del Sur), con cámara frontal de 16 megapíxeles de resolución, a los especímenes in vivo.
Una vez herborizados fueron depositados en la colección micológica del Herbario del Instituto de Ecología y Sistemática (HAC, acrónimo según Thiers, 2021), perteneciente al Ministerio de Ciencia, Tecnología y Medio Ambiente de Cuba. Para la descripción de las características macro y microscópicas e identificación de la especie, se siguieron los criterios de Lowy (1959, 1971), McNabb (1964), Niveiro y Popoff (2011) y Chen et al. (2020). Para la descripción de los colores se utilizó la terminología propuesta por Kornerup y Wanscher (1978).

\section{Resultados}

\section{Taxonomía}

\section{Basidiomycota}

\section{Agaricomycotina}

\section{Agaricomycetes}

\section{Auriculariomycetidae}

Auriculariales

\section{Exidiaceae}

Pseudohydnum gelatinosum (Scop.) P. Karst., Not. Sällsk. Fauna et Fl. Fenn. Förh. 9: 374. 1868. MB\#120525. Fig. 2.

$\equiv$ Hydnum gelatinosum Scop., Fl. Carniol., Ed. 2 (Wien) 2: 472. 1772.

Basidiomas gelatinosos, lateralmente estipitados, imbricados; píleo de dimidiado a flabeliforme, 15-25 × 20$35 \mathrm{~mm}$; superficie del píleo lisa a finalmente papilada, blanco grisácea (1B1) en estado fresco, amarillenta a amarillo grisácea al secar (4B3); margen entero; contexto delgado, gelatinoso, 5-8 mm de grosor, grisáceo (4B2); himenóforo hidnoide; acúleos cónicos, blancos (4A1), 3.3-8 ×1.3-4 mm; estípite corto, concoloro con la superficie del píleo, 4-10 $\mathrm{mm}$ de longitud; basidios globosos, hialinos, 10-14.6 × 7.5$11 \mu \mathrm{m}$, divididos longitudinalmente $\mathrm{y}$ formados por cuatro 


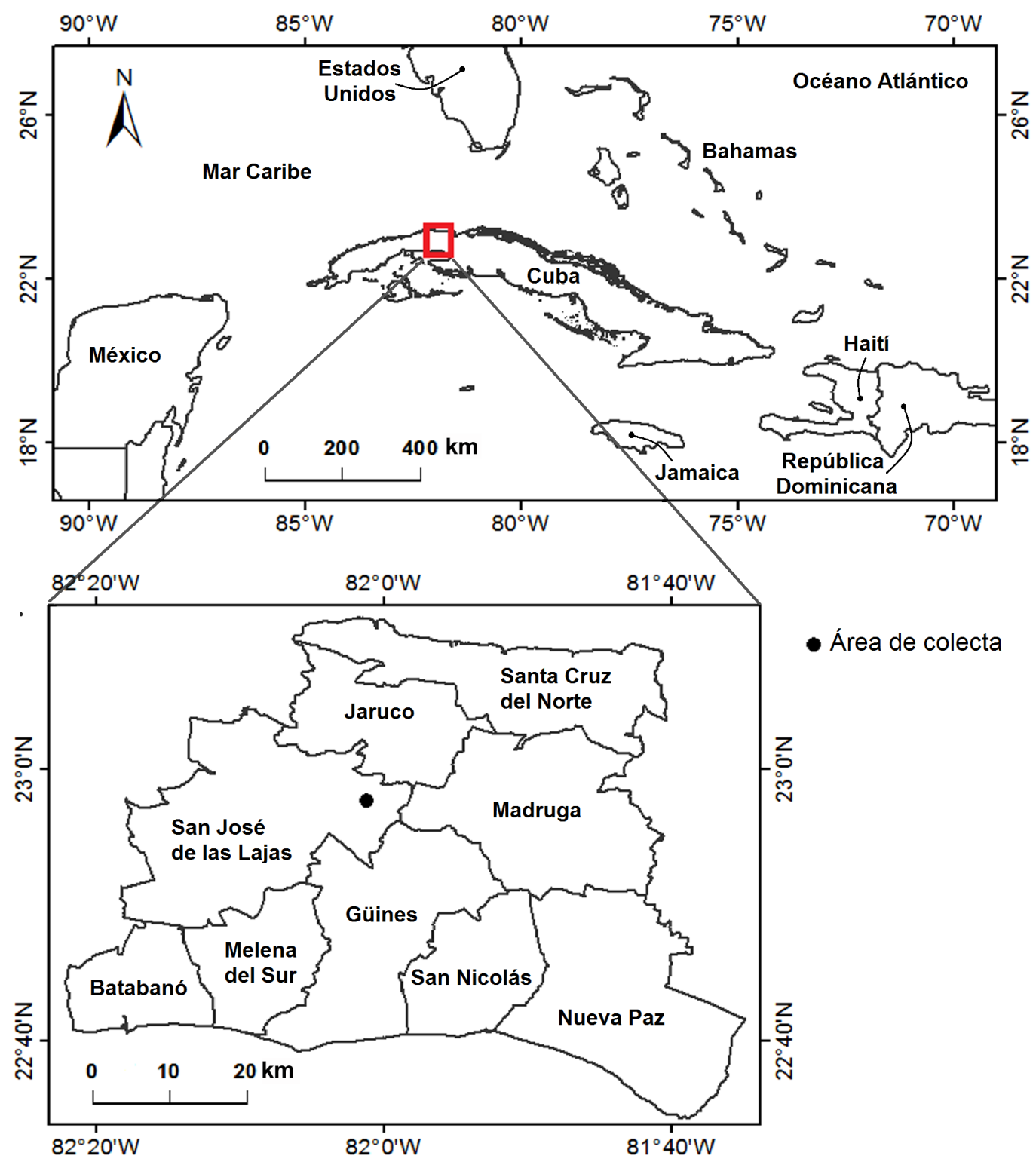

Figura 1: Localidad de colecta de Pseudohydnum gelatinosum (Scop.) P. Karst., en Mayabeque, Cuba.

células, con cuatro esterigmas cilíndricos, 5-23 × 1.5-3 $\mu \mathrm{m}$; basidiosporas de subglobosas a elipsoides, hialinas, lisas, inamiloides, indextrinoides, 6-7.8(-9) $\times(4-) 4.4-4.9(-5.3)$ $\mu \mathrm{m}, \mathrm{Q}=1.1-1.3, \mathrm{Qm}=1.5$; germinación no observada; hifas fibuladas, ramificadas, de hialinas a amarillentas, 2-3 $\mu \mathrm{m}$ de diámetro.

Ejemplares estudiados: CUBA. Mayabeque, municipio San José de las Lajas, en las cercanías del kilómetro 4.5 de la carretera al poblado de Casiguas, 148 m s.n.m., $22^{\circ} 57^{\prime} 51.0^{\prime \prime} \mathrm{N}, 82^{\circ} 01^{\prime} 11.1^{\prime \prime} \mathrm{O}$, vegetación secundaria, sobre tronco seco de Cupania glabra Sw. (Sapindaceae), 18.XI.2018, L. Castro S.n. (HAC 11571).

\section{Discusión}

Pseudohydnum gelatinosum podría confundirse con las otras dos especies del género debido a la estrecha relación morfológica entre ellas (Chen et al., 2020). Morfológicamente, $P$. gelatinosum y $P$. translucens son similares, debido a la coloración blanquecina del píleo en ambas 

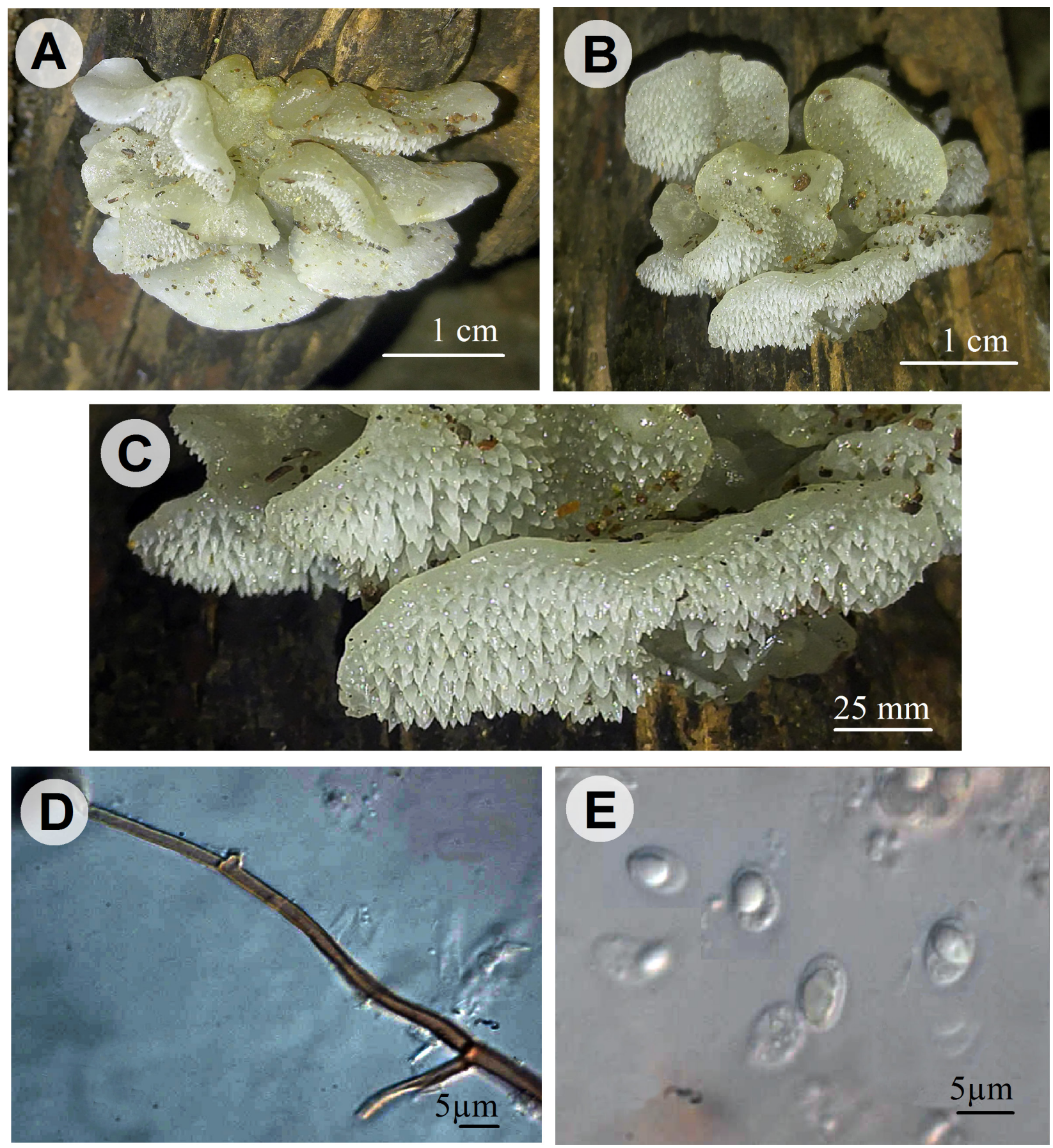

Figura 2: Pseudohydnum gelatinosum (Scop.) P. Karst. A. superficie del píleo; B. himenóforo; C. dientes del himenóforo; D. hifa; E. basidiosporas.

especies; no obstante, en esta última el contexto es más delgado $(1 \mathrm{~mm})$ y las esporas de menores dimensiones (4 $\times 5 \mu \mathrm{m})$, como refiere Lloyd (1925), lo que argumenta la separación.

En comparación con P. brunneiceps, P. gelatinosum presenta basidiosporas con rangos que se traslapan, pero otros caracteres diagnósticos las separan claramente, coincidiendo con Chen et al. (2020). Pseudohydnum gelatinosum presenta píleo blanco grisáceo, contexto de 5-8 $\mathrm{mm}$ de grosor y basidiosporas hialinas, mientras que $P$. brunneiceps posee píleo parduzco, contexto de menor grosor (1-2 $\mathrm{mm})$ y basidiosporas amarillo parduzcas. De igual 
modo, los resultados moleculares obtenidos por Chen et al. (2020) indican una separación entre estos dos taxones, pues los dividen en linajes independientes.

A pesar de que Pseudohydnum gelatinosum se distribuye mundialmente en cuatro continentes (Far y Rossman, 2021; GBIF, 2021), los registros se concentran en las regiones templadas del hemisferio norte, como Europa y Norteamérica. En el Caribe insular, la presencia de la especie se restringía a Puerto Rico (Minter et al., 2001) y Jamaica (GBIF, 2021), con un registro en cada país, por lo que no es frecuente en esta región.

Con el presente trabajo, el género Pseudohydnum se suma a la lista de basidiomicetos con himenóforo hidnoide citados para Cuba y por consiguiente es el primer registro de la especie $P$. gelatinosum para el país; con lo cual se amplía el área de distribución de ésta en el Caribe insular. De igual modo, se reafirma la importancia de continuar realizando muestreos micológicos, además de proponer su evaluación en futuras versiones de la Lista Roja de los Hongos de Cuba, debido a su escasa presencia en el país y otras islas del Caribe.

\section{Contribución de autores}

LC concibió y diseñó el estudio. LC y MC tomaron las fotografías y examinaron los materiales. LC, MC y YA contribuyeron a la escritura del manuscrito, su discusión, revisión y aprobación de la versión final del mismo.

\section{Agradecimientos}

A los editores y árbitros anónimos de la revista Acta Botanica Mexicana, que contribuyeron a perfeccionar este manuscrito.

\section{Financiamiento}

Este estudio fue apoyado por el proyecto "Implementación de la Estrategia de Conservación de la Diversidad Fúngica en Cuba" (código de proyecto: P211LH005-047), aprobado por el Programa Nacional de la Agencia de Medio Ambiente "Uso sostenible de los componentes de la Diversidad Biológica en Cuba" y liderado por la Subdirección de Micología del Instituto de Ecología y Sistemática (CITMA).

\section{Literatura citada}

Bera, M., S. Paloi, A. K. Dutta, P. Pradhan, A. Roy y K. Acharya. 2018. Contribution to the Macromycetes of West Bengal, India: 23-27. Journal of Threatened Taxa 10(9): 12270-12276. DOI: https://doi.org/10.11609/jott.3875.10.9.12270-12276

Chen, Y. L., M. S. Su, L. P. Zhang, Q. Zou, F. Wu, N. K. Zeng y M. Liu. 2020. Pseudohydnum brunneiceps (Auriculariales, Basidiomycota), a new species from Central China. Phytotaxa 441(1): 87-94. DOI: https://doi.org/10.11646/ phytotaxa.441.1.8

Dai, Y., Z. L. Yang, B. K. Cui, C. J. Yu y L. W. Zhou. 2009. Species Diversity and Utilization of Medicinal Mushrooms and Fungi in China. International Journal of Medicinal Mushrooms 11(3): 287-302. DOI: https://doi.org/10.1615/ IntJMedMushr.v11.i3.80

ESRI. 2013. ArcGIS Desktop and Spatial Analyst Extension: Release ver. 10.2. Environmental Systems Research Institute. Redlands, USA.

Far, D. F. y A. Rossman. 2021. Fungal Database. U.S. National Fungal Collections, The Agricultural Research Service (ARS), the United States Department of Agriculture (USDA). http://nt.arsgrin.gov/fungaldatabases (consultado noviembre de 2021).

GBIF. 2021. Global Biodiversity Information Facility. GBIF Occurrence Download. Copenhague, Dinamarca. DOI: https://doi.org/10.15468/dl.qdja2r

Kornerup, A. y J. H. Wanscher. 1978. Methuen handbook of colour. 3a ed. Eyre Methuen. London, UK. 252 pp.

Lloyd, C. G. 1925. Mycological Notes 75. Mycological Writings 7: 1349-1364.

Lowy, B. 1959. New or Noteworthy Tremellales from Bolivia. Mycologia 51(6): 840-850. DOI: https://doi.org/10.1080/0 0275514.1959.12024864

Lowy, B. 1971. Tremellales. Flora Neotropica 6: 1-153.

McNabb, R. F. R. 1964. New Zealand Tremellales-I. New Zealand Journal of Botany 2(4): 403-414. DOI: https://doi.org/10.10 80/0028825X.1964.10428761

Minter, D. W., M. Rodríguez y J. Mena. (eds.). 2001. Fungi of the Caribbean. An annotated checklist. UDMS Publishing. Glasgow, UK. 946 pp.

Niveiro, N. y O. Popoff. 2011. Pseudohydnum gelatinosum (Tremellales, Basidiomycota) en las Yungas Argentinas. 
Boletín de la Sociedad Argentina de Botánica 46(3-4): 223226.

Olson, D. M., E. Dinerstein, E. D. Wikramanayake, N. D. Burgess, G. V. N. Powell, E. C. Underwood, J. A. D’amico, I. Itoua, H. E. Strand, J. C. Morrison, C. L. Loucks, T. F. Allnutt, T. H. Ricketts, Y. Kura, J. F. Lamoreux, W. W. Wettengel, P. Hedao y K. Kassem. 2001. Terrestrial Ecoregions of the World: A New Map of Life on Earth. BioScience 51(11): 993-938. DOI: https://doi. org/10.1641/0006-3568(2001)051[0933:TEOTWA]2.0.CO;2
Raymundo, T., M. Contreras, S. Bautista-Hernández, R. DíazMoreno y R. Valenzuela. 2012. Hongos tremelloides del bosque Las Bayas, municipio de Pueblo Nuevo, Durango, México. Polibotánica 33: 85-103.

Thiers, B. 2021 continuously updated. Index Herbariorum, a global directory of public herbaria and associated staff. New York Botanical Gardens Virtual Herbarium. New York, USA. http://sweetgum.nybg.org/science/ih/ (consultado octubre de 2021). 